\title{
A Review of the Automation of the Czochralski Crystal Growth Process
}

\author{
J. Winkler ${ }^{a, *}$, M. NEUBERT ${ }^{b}$ AND J. Rudolph ${ }^{c}$ \\ ${ }^{a}$ Institut für Regelungs- und Steuerungstheorie, Technische Universität Dresden, Germany \\ ${ }^{b}$ Leibniz-Institut für Kristallzüchtung, Berlin, Germany \\ ${ }^{c}$ Lehrstuhl für Systemtheorie und Regelungstechnik, Universität des Saarlandes, Saarbrücken, Germany \\ On the occasion of the centennial of the invention of the Czochralski crystal growth process by the Polish \\ scientist Jan Czochralski, a review of selected strategies for the automatic control of this process is given. This \\ review provides a sketch of the fundamental challenges of controlling the Czochralski process and the basic concepts \\ of feedback control. Both early and modern approaches to the control of the Czochralski process are described. \\ The discussion focuses on questions related to feed-forward control, feedback control, and state estimation. The \\ presented methods rely on simple mathematical process models in contrast to the finite element model-based \\ approaches typically used in crystal growth process design and analysis. Such mathematical models motivate both \\ the structure and parameters of the chosen controller. A comprehensive list of references to background literature \\ on this topic completes this survey.
}

DOI: 10.12693/APhysPolA.124.181

PACS: 81.10.Fq, 81.10.Aj, 02.30.Yy

\section{Introduction}

This contribution presents an overview about the challenges as well as about past and present strategies for automatic control of the Czochralski $(\mathrm{Cz})$ crystal growth process, one of the most important growth technologies used in industrial crystal growth. The demand for crystals of well defined structural, chemical and electrical properties produced with a maximum rate of yield and reproducibility was - and still is - the driving force to develop highly sophisticated automatic control systems. In this growth method, as it is mostly practiced nowadays, the crystals are grown freely from the melt, i.e., there are no shaping devices ensuring a constant or well defined diameter which is mainly important in matters of technological requirements. (Some variants of the $\mathrm{Cz}$ process used a so-called coracle - a floating diameter-defining aperture made from sintered silicon nitride - to ensure a stable thermal regime around the growing crystal and hence to stabilize a constant diameter, cf. [1].) The better it meets the desired diameter and the better its constancy, the less material has to be cut off after growth. On the other hand, strong diameter changes influence the structural properties of the crystal [2-6].

Not only the diameter, also other quantities like the crystal growth rate are of special importance. The growth rate directly influences the properties of the growing crystal. It correlates with the amount of imperfections, like polycrystalline growth, twins, dislocations as well as the amount of native point defects, residual impurities and that of intentionally introduced dopants. Consequently, for process technology there is a huge interest

*corresponding author; e-mail: Jan.Winkler@tu-dresden.de in growth rate control also, especially in silicon crystal growth where the relation between growth rate and thermal gradients at the interface is of great importance in order to ensure an extremely low content of point defects [7-12].

No matter which control strategy is chosen to address these issues, the $\mathrm{Cz}$ system is an extremely challenging control object giving control engineers a headache for decades now. These issues are subject of Sect. 2 .

When designing a control system the first task is to characterize the dynamic relations between the manipulated and the controlled variables. Based on these results a control structure is chosen. A large variety of approaches are provided by modern control theory. Such a control system does not only consist of a feedback controller, but also on equipment realizing the feed-forward control, the reference trajectory planning and the reconstruction of not directly measured variables. Different levels of mathematical complexity, questions about linear or nonlinear control design, robustness and real-time capability make things more difficult. Section 3 gives an introduction to the basic terms and approaches used in control technology as required for the $\mathrm{Cz}$ process.

When the $\mathrm{Cz}$ process was introduced into industrial germanium growth $[13,14]$ the weight measurement of the crucible or of the growing crystal to control the heater power [15] was used. In contrast to that Patzner et al. used an optical sensor manipulating the pull rate for silicon [16]. Nowadays, diameter control has been developed for a variety of different materials and system configurations, where many complex problems have been identified such as materials with hard to control conditions that resulted either in high dislocation densities or large growth and shape variations, as well as the challenges in scale up of the process for larger crystal sizes [8, 11, 17-22]. Other 
examples are the use of a liquid encapsulant [23, 24] introducing time delays into the process [25, 26] or more sophisticated variants of the $\mathrm{Cz}$ process, like the vapor pressure controlled Czochralski ( $\mathrm{VCz}$ ) method [27] which intentionally leads to reduced axial and radial temperature gradients making diameter control even more difficult $[28,29]$. More recently, the focus of control design has been extended to maintain additional degrees of freedom which affect crystal quality as well as crystal shape during the neck, shoulder and tail sections in addition to the main body [30, 31]. In Sect. 4 selected strategies will be discussed.

\section{Specific characteristics of the $\mathrm{Cz}$ process}

The aim of process analysis is to gain insight into its dynamical behavior using powerful methods provided by systems theory. For this purpose a wide range of analysis methods exist. First analysis of the principal system behavior in order to improve diameter feedback control were presented by Bardsley et al. in the 70's [32-36]. Some of the main results are summarized in the following.

\subsection{Meniscus region}

The most important region in the Czochralski crystal growth is the meniscus, the interconnection between melt and crystal, cf. Fig. 1. The meniscus and its shape result from gravitational forces and surface tension. At its upper end the so called phase boundary, or interface, is located where crystallization takes place. Thereby heat of fusion (latent heat) is released. Because the crystal is pulled upward into colder regions of the furnace a temperature gradient is established, which leads to a heat flow by conduction from the hot interface into the colder crystal [18]. By this mechanism crystallization is maintained throughout the growing process.

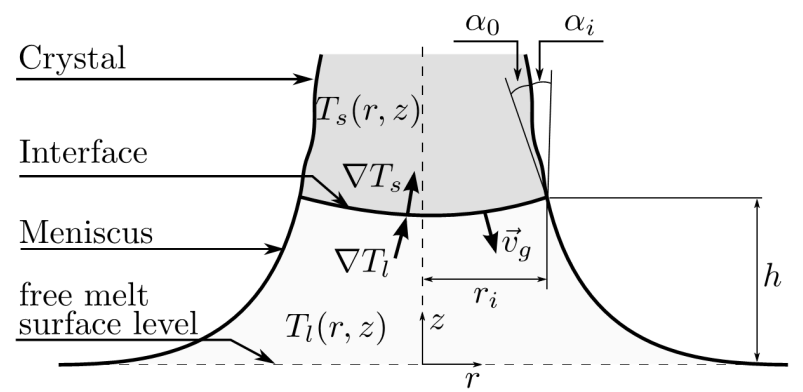

Fig. 1. Sketch of the interface region with important physical quantities.

The amount of heat transported into the crystal consists of two components: the amount of heat transported from the meniscus region into the interface with radius $r_{\mathrm{i}}$ and the amount of heat released by crystallization. In general, the following relation holds for the vector $\boldsymbol{v}_{\mathrm{g}}$ of the growth rate along the phase boundary described by $z=\mathcal{Z}(r), r \in\left[0, r_{\mathrm{i}}\right]$ :

$$
\boldsymbol{v}_{\mathrm{g}}(r, \mathcal{Z}(r))=\frac{1}{\rho_{\mathrm{s}} \Delta H_{\mathrm{f}}}\left(\lambda_{\mathrm{m}} \nabla T_{\mathrm{m}}(r, \mathcal{Z}(r))\right.
$$

$$
\left.-\lambda_{\mathrm{s}} \nabla T_{\mathrm{s}}(r, \mathcal{Z}(r))\right), \quad r \in\left[0, r_{\mathrm{i}}\right] .
$$

In this equation the specific latent heat is denoted by $\Delta H_{\mathrm{f}}$, the heat conductivities of the solid and the melt are given by $\lambda_{\mathrm{s}}$ and $\lambda_{\mathrm{m}}$, respectively, and $T_{\mathrm{s}}(r, z)$ and $T_{\mathrm{m}}(r, z)$ represent the temperature of the solid and the melt. The density of the crystal at the interface (at melting temperature $T_{\mathrm{i}}$ ) is given by $\rho_{\mathrm{s}}$. A necessary condition for growth is that the growth rate is positive, i.e. the heat flux is directed from the melt into the crystal.

Changes in the temperature gradients on the melt or solid side of the phase boundary lead to an immediate change of the growth rate, as can be easily seen from Eq. (1). On the one hand, a local change of the growth rate results in a deformation of the phase boundary and in a change of the crystal diameter if this deformation is located at the rim of the interface. On the other hand, a change in interface geometry again initiates a change in heat transport. Furthermore, the shape of the meniscus strongly depends on the radius $r_{\mathrm{i}}$ of the crystal at the interface as well as on the growth angle $\alpha=\alpha_{0}+\alpha_{\mathrm{i}}$ with the equilibrium growth angle $\alpha_{0}$ and the crystal slope angle $\alpha_{\mathrm{i}}[37-39]$, cf. Fig. 1 . This means that changes in the meniscus shape initiated by changes in the geometry result in an immediate change of the heat balance in this region, with the consequences described above. This consideration leads to the conclusion that the demand of growing crystals of well defined shape requires exact control of the thermal conditions in the interface region. Without any precise measurements characterizing the state of this region this is an overwhelming task. An approach trying to realize such a direct control by blowing inert gas around this region is presented by Brice [40]. However, usually any measurements available at the process are more or less distant to this region complicating the control of the process.

\subsection{Measurement issues}

The quantities which one would like to control are the crystal diameter and the crystal growth rate. Unfortunately, these quantities cannot be directly measured. As an alternative the force acting on the pulling rod (or the crucible) [15] or the diameter of a bright ring on the meniscus resulting from reflections of the hot glowing heaters [41] are available. For example, in the liquid encapsulated Czochralski (LEC) process the crystal diameter is not directly measured because of a boron oxide layer covering the melt. Hence, in this process the weighing method is used.

The idea behind the weighing technique is that - at a first glance - the rate of change of the force acting on the load cell might be proportional to the crystal cross-section area at the growing interface. This can be evaluated by a controller. However, the measured force is also influenced by forces resulting from the meniscus $[34,42]$ making the correct interpretation of the signal more complicated. Furthermore, if the density of the solid is smaller than the density of the liquid, a well known anomaly comes into play which consists of the 
fact that, e.g., an increase in the differential weight gain signal does not necessarily reflect an increase of the crystal diameter. The reason for this effect is that an increasing crystal diameter first results in a decrease of the meniscus-height and of its volume (cf., Fig. 2, middle). Since the density of the melt is larger than the density of the solid, a decreasing meniscus-volume makes the differential weight gain signal decreasing at first although the crystal diameter is increasing (cf., Fig. 2, bottom). For materials which do not completely wet their solids, i.e., $\Theta_{0} \neq 0$, a similar effect can be observed since an increase in crystal diameter $\left(\alpha_{\mathrm{i}}>0\right)$ leads to a reduction of the vertical component of the surface tension acting on the crystal (its vertical component is proportional to $\left.\cos \left(\Theta_{0}+\alpha_{\mathrm{i}}\right)\right)$. Unfortunately, all commonly grown semiconducting materials show this effect.

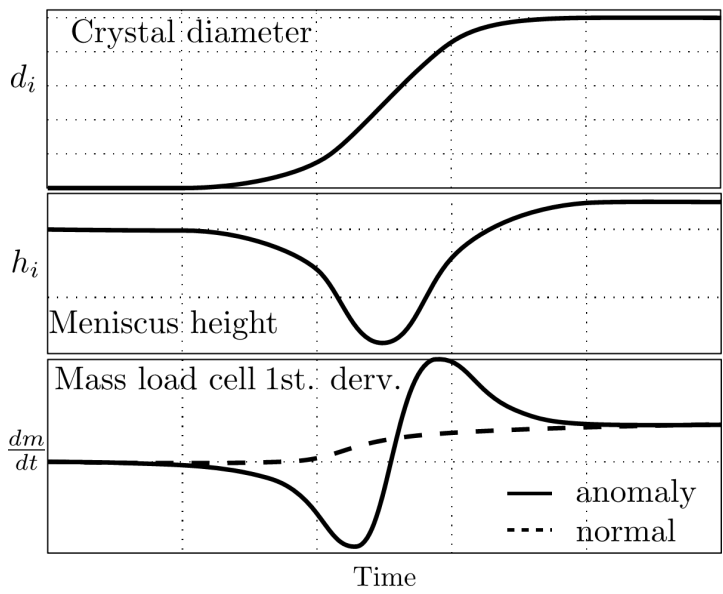

Fig. 2. A slight change of the crystal diameter (top) results in a temporarily break-in of the differential weight gain signal (bottom). The reason for that is the reduction of the meniscus height during the diameter transfer (middle). After [34].

The anomaly also leads to crucial problems during the transfer from the shoulder into cylindrical growth, cf., Fig. 3 (top): a sudden increase of the derivative of the force acting on the load cell with respect to time is observed just before the crystal diameter reaches the cylinder. This behavior can be explained by the fact that the shape of the meniscus changes significantly at this phase of the process [30].

More detailed discussions with respect to this subject can be found in [42-44]. The strength of the effect depends on several factors discussed in detail in [35]. Especially the growth rate is of great importance. Roughly speaking it can be summarized that the lower the pulling speed the stronger the effect. Measurement of the diameter of the bright meniscus ring, as it is widely used in silicon growth, is affected by a similar anomaly [17].

Yet another factor limiting the weighing technique is the diameter of the crystal body. The larger it is the stronger the load cell measuring the force has to be. How-

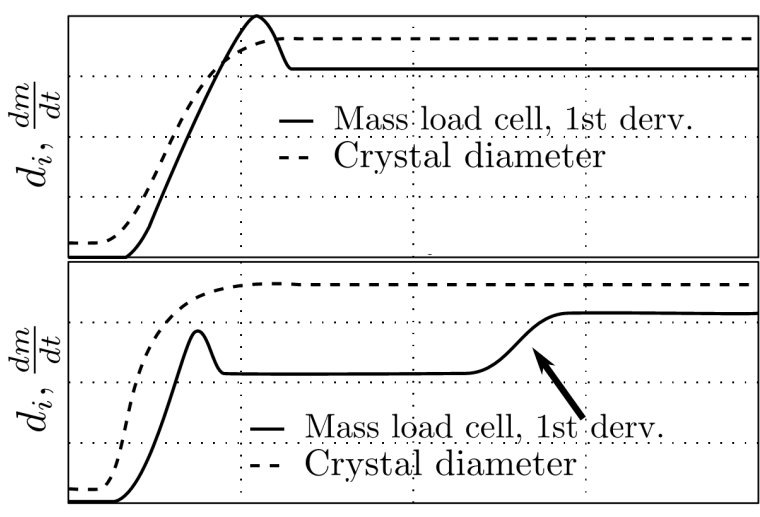

Time

Fig. 3. Top: increase of the derivative of the differential weight gain signal before the crystal reaches the phase of cylindrical growth. Bottom: in case of the LEC-process an "aftershock" occurs in the differential weight gain signal when the crystals emerge from the boron oxide layer. The dashed curves represent the corresponding diameter trends.

ever, the stronger the load cell the more inaccurate it becomes for small loads. This is problematic especially during the initial phase of the process.

\subsection{Batch character and time delays}

The $\mathrm{Cz}$ process is a so-called batch process. This means that it does not have a steady state operating point. This mainly results from the fact that the whole system configuration changes during the growth: while in the beginning one has a crucible filled with melt, one has a nearly empty crucible and a crystal at the end of the process. The falling melt level leads to an ever-changing heat entry from the heaters into the system. Thus, the process dynamics changes heavily throughout the process. Understanding the influence of these variations on the process dynamics provides a foundation for developing a robust process control system. Detailed discussions on these subjects can be found in $[2,17,45,46]$.

In case of LEC growth, the influence of the boron oxide layer comes into play. One has to take into account the buoyancy as well as the time delay introduced by the emerging crystal, resulting in additional dynamical effects. For example, Fig. 3 (bottom) shows an "aftershock" occurring in the differential weight gain signal when the crystal begins to emerge from the boron oxide layer. These effects are discussed in [25, 47, 48] and must be carefully considered during control system design.

\subsection{Modeling issues}

Today, modeling and computer simulation play an important role in crystal growth process design as well as in control applications. For example, whenever no direct access to certain quantities in a process is possible dynamic mathematical models are used to establish some kind of connection between the measured and the not measured quantities in order to reconstruct the values of the latter ones. However, since the dynamics of the 
process is mainly driven by complex nonlinear radiative, conductive, and convective heat transport phenomena, it cannot be fully captured through a linear model $[2,8]$. Especially the fact that the warm regions of this process are found at the bottom while the colder ones are located at the top of the plant leads to strong convection phenomena which cannot be neglected. Hence, one has to switch to nonlinear multi-dimensional time dependent models which are more complicated to handle. Above all, the $\mathrm{Cz}$ process is a system with free boundaries (the solid-liquid interface and the crystal shape) making it even more complicated to solve the underlying dynamic equations. Many unknown physical parameters and the complex structure of the inner assemblies worsen the situation. Here, from a control technological point of view, one has to find a balance between accuracy and real time capability of the model $[21,30]$.

\subsection{Stability}

An important matter in control theory is the question if the system under consideration is stable or not, since unstable systems have a limitation to achievable control performance. When discussing this subject it is very important which type of model is used for stability analysis and which physical effects are included in the model: Surek's initial stability analysis was only of the capillary problem, i.e., for the meniscus region [49]. He came to the conclusion that the capillary system is unstable. However, if the heat fluxes are taken into account, one comes to a different conclusion, depending on the assumptions made during modeling. Thus, Refs. [39, 50-52] as well as [53] show stable behavior of the growth system under certain operating conditions. Nevertheless, Refs. [54] and [55] show that the system is unstable for the cases they evaluated, even if the thermal effects are included in the analysis.

\section{Components of a control system}

The intention of this section is to introduce some of the basic concepts used in control technology as far as they are required for an understanding of the numerous strategies for control of the $\mathrm{Cz}$ process. For this purpose the essential components of a control system are shortly summarized (cf. Fig. 4) followed by a discussion of the way mathematical models are used for control purposes, which differs from the way they are used for thermal analysis or plant and process design.

\subsection{Reference trajectory generator}

Depending on time or crystal length a reference trajectory generator calculates reference values for crystal radius and growth rate or other useful quantities. In the simplest case the trajectory generator holds a fixed value, often called the setpoint or "hand value". Planning of trajectories has to consider the physical properties of the system in order to avoid, e.g., impossible values for the control inputs. Furthermore, one cannot request physically impossible behavior from the system, such as steps in any of the system quantities or their derivatives.

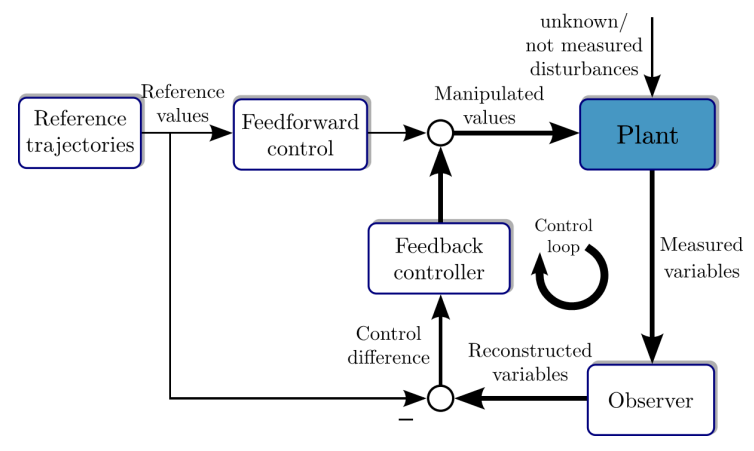

Fig. 4. Sketch of a modern feedback control loop.

It may be useful to reschedule the reference trajectories during growth to guarantee smooth behavior of the controller.

\subsection{Feed-forward controller}

The feed-forward control calculates the manipulated values (like heater power or crystal pulling rate) from the reference values generated by the trajectory generator. This can be done empirically or by means of a mathematical model describing the system behavior. The latter strategy is also known as the solution of the inverse problem, in contrast to the forward problem which consists of the solution of a dynamic model depending on given initial values and given trajectories for the inputs. For example, one has a system with the manipulated variable $u \in \mathbb{R}$, the output variable (to be controlled) $y \in \mathbb{R}$ and two internal states $x_{1}, x_{2} \in \mathbb{R}$. Its dynamics is captured by the following model:

$$
\begin{aligned}
& \dot{x}_{1}=x_{2}, \\
& \dot{x}_{2}=x_{1}^{2}+\left(x_{2}+2\right) u, \\
& y=x_{1} .
\end{aligned}
$$

From this model a model based feed-forward control can be easily calculated. Solving Eq. (2b) for $u$ one obtains

$$
u=\frac{\dot{x}_{2}-x_{1}^{2}}{x_{2}+2} \text {. }
$$

Now, one would like to steer the system, namely the output $y$, along a reference trajectory $t \mapsto y_{\text {ref }}(t)$. This reference trajectory is assumed to be twice continuously differentiable, i.e., $\dot{y}_{\text {ref }}(t), \ddot{y}_{\text {ref }}(t)$ exist. Since according to system (2) one has $x_{1}=y, x_{2}=\dot{x}_{1}=\dot{y}$, and $\dot{x}_{2}=\ddot{y}$ the resulting reference trajectory for the manipulated variable $u$ reads (cf. Eq. (3)):

$$
u_{\mathrm{ref}}=\frac{\ddot{y}_{\mathrm{ref}}-y_{\mathrm{ref}}^{2}}{\dot{y}_{\mathrm{ref}}+2} .
$$

Using the values from the feed-forward control one might be able to steer the system along its reference trajectory if the system is stable, only small perturbations are acting on the system, and the model is accurate enough.

\subsection{Feedback controller}

The feedback controller compensates for disturbances acting on the process. Furthermore, it is responsible for 
correction of modeling errors resulting in inaccuracies of the feed-forward control. This is achieved by feedback of the measured variable (or its estimate) and a correction of the input calculated by the feed-forward control.

It is common practice to realize feedback control by means of a PID controller. The PID controller processes a deviation $e(t)$ between the desired and the real value of the variable to be controlled in order to calculate a change $\Delta u(t)$ of the manipulated variable

$$
\Delta u(t)=K_{\mathrm{P}} e(t)+K_{\mathrm{I}} \int_{t_{0}}^{t} e(\tau) \mathrm{d} \tau+K_{\mathrm{D}} \frac{\mathrm{d} e}{\mathrm{~d} t}(t) .
$$

The dynamic behavior of the PID controller is determined using the three real constants $K_{\mathrm{P}}, K_{\mathrm{I}}$, and $K_{\mathrm{D}}$. It is an implementation of the following natural assumptions: (i) The larger the control deviation, the larger one has to counter steer, an implication which is covered by the proportional part $K_{\mathrm{P}} e(t)$. (ii) The longer the control deviation is pending, the larger the manipulated variable has to be changed in order to reduce it. This behavior is realized by the integral part parameterized using the factor $K_{\mathrm{I}}$, the second term in Eq. (5). (iii) The faster the control deviation changes, the stronger one has to counteract - this is covered by the third term in Eq. (5) the differential part with parameter $K_{\mathrm{D}}$.

The PID controller has several advantages: its operating principle is simple, it can be easily implemented, if certain conditions are hold it can be adjusted using some simple rules, it is suitable for a wide range of technological systems found in practice. However, there are some drawbacks: it is a linear controller. This means that its parameterization is adjusted for a certain operating point of the process. If one does not have an operating point (as it is the case in the Czochralski growth, cf. Sect. 2.3), parameters have to be continuously changed during the growth. The same holds for an intentionally initiated change of the operating point during the process. The performance of PID controllers is limited if the system shows nonlinear behavior. The reason for this is the fact that a change of the manipulated variable is processed nonlinearly in the process resulting in "unexpected" reactions (in comparison to a linear one) of the system. Situation worsens if the system contains time delays. In this case a reaction of the system to a change of the manipulated variable appears delayed by the delay time $\tau$. This may result in an undamped increasing integral of the PID algorithm (cf. Eq. (5)). In the Czochralski crystal growth the LEC process with its boron-oxide layer covering the melt belongs to the class of delay systems. In summary, a PID controller does not necessarily fit the dynamics of the system, especially if the order of the system is larger than two. Therefore, it may be very time consuming to find appropriate parameters. Consequently, the performance of diameter control based on the use of linear PID controllers, the parameters of which are chosen heuristically, is limited [8, 43, $56,57]$.
Fortunately, theory and application of control systems have been developed very rapidly in the last decades. Increasing capacity of micro-controllers and programmable logic controllers (PLC) combined with powerful mathematical control design methods prepared the ground for the use of sophisticated model based controllers in practice. A model based controller uses a mathematical model of the system, i.e., its structure and parameters fit to the system. This means that the controller can be used in a wide range of operating points and reflects the nonlinear system characteristics as well as time delays. In addition, parameterization effort is dramatically reduced. Anyhow, although model based control sounds promising, such controllers do show good performance only if the underlying model matches the structure and the parameters of the system appropriately. It will be shown in the following sections that this fact is the crucial point when trying to enhance the capability of conventional control systems.

In order to make the strategy of model based control more clear, the design of a simple nonlinear feedback controller based on a lumped parameter model is sketched. Again, the system (2) of the previous section is used. One wants to make the output $y$ follow a reference trajectory $y_{\text {ref }}(t)$. For this one defines the tracking error $y-y_{\text {ref }}$. Now the controller is designed in such a way that the tracking error satisfies the linear second order differential equation

$$
\left(\ddot{y}-\ddot{y}_{\text {ref }}\right)+k_{1}\left(\dot{y}-\dot{y}_{\text {ref }}\right)+k_{0}\left(y-y_{\text {ref }}\right)=0
$$

with $k_{0}, k_{1}>0$.

The values of $y_{\text {ref }}, \dot{y}_{\text {ref }}$, and $\ddot{y}_{\text {ref }}$ are defined by the reference trajectory. If one desires the dynamics of the tracking error to satisfy Eq. (6) the second time derivative of the output $y$ must behave as

$$
\ddot{y}=\ddot{y}_{\text {ref }}-k_{1}\left(\dot{y}-\dot{y}_{\text {ref }}\right)-k_{0}\left(y-y_{\text {ref }}\right) .
$$

This can be achieved by a proper choice of the input $u$ because by inserting the right hand side of (7) in the equation used for calculation of $u$ (cf. Eq. (3)) one obtains

$$
\begin{aligned}
u & =\frac{\dot{x}_{2}-x_{1}^{2}}{x_{2}+2}=\frac{\ddot{y}-y^{2}}{\dot{y}+2} \\
& =\frac{\ddot{y}_{\mathrm{ref}}-k_{1}\left(\dot{y}-\dot{y}_{\mathrm{ref}}\right)-k_{0}\left(y-y_{\mathrm{ref}}\right)-y^{2}}{\dot{y}+2} .
\end{aligned}
$$

This equation determines the manipulated variable $u$ in such a way that the tracking error satisfies the given error dynamics (6). The coefficients $k_{0}, k_{1}$ in Eq. (6) are the controller parameters, the real value inputs are $y, \dot{y}$, and the desired value inputs are $y_{\text {ref }}, \dot{y}_{\text {ref }}, \ddot{y}_{\text {ref }}$. The coefficients $k_{0}, k_{1}$ define the dynamical behavior of the tracking error, i.e., the way the error converges to zero (e.g. fast with an overshooting or slowly creeping).

\subsection{State estimator}

Finally, it is not always possible to measure the controlled variable (in case of the Czochralski method the radius) directly. In that case one can reconstruct this variable from the measured variable by means of a (possibly nonlinear) observer if a suitable model of the sys- 
tem is available and the system is observable (a system property discussed later).

In order to overcome this difficulty one has to reconstruct the crystal radius from the measured force. This can be done by a so-called "observer". It is based on the following idea. Assume a mathematical model

$$
\dot{x}(t)=f_{1}(x(t), u(t)), y(t)=f_{2}(x(t), u(t)), x(0)=x_{0}
$$

of a process with $(x(t), u(t)) \in \mathbb{R}^{n} \times \mathbb{R}^{r}$ and $y(t) \in \mathbb{R}^{m}$. If some components of $x$ are not directly measured, one might implement a "copy"

$$
\dot{\hat{x}}(t)=f_{1}(\hat{x}(t), u(t)), \hat{y}(t)=f_{2}(\hat{x}(t), u(t)), \hat{x}(0)=\hat{x}_{0}
$$

of the system model in a computer program. The trajectories of the manipulated variables $u$ acting on the real system are fed into this computer program, too. Then one has access to all components of $\hat{x}$. Since the initial values of $\hat{x}$ are not known, i.e., $\hat{x}_{0} \neq x_{0}$, and because of model inaccuracies as well as disturbances acting on the system, generally the values calculated for $\hat{x}(t)$ will not be equal to the real values $x(t)$. However, under certain circumstances it is possible to inject the error $\hat{y}-y$ between the estimated value $\hat{y}$ and the measured value $y$ in such a way that the difference between the calculated value $\hat{x}$ and the real value $x$ will converge to zero as time advances [58]. In this case the system

$$
\begin{aligned}
& \dot{\hat{x}}(t)=h(\hat{x}(t), u(t), \hat{y}(t)-y(t)), \quad \hat{x}(0)=\hat{x}_{0}, \\
& \hat{y}(t)=f_{2}(\hat{x}(t), u(t))
\end{aligned}
$$

is called an observer.

Design and dimensioning of the injection of the error related to the measured variable is the key task when developing an observer. However, it is important to know if it is possible to reconstruct a quantity from the measured variable at all. This property is commonly referred to as "observability".

3.5. Modeling in crystal growth analysis and control

A short comparison on how models are used in automatic control in contrast to numerical approaches in crystal growth will be given here, since between both fields the approaches and requirements differ quite a bit.

A mathematical model which takes the spatial distribution as well as the time dependence of material parameters into account leads to a system of coupled partial differential equations with the corresponding initial, boundary, and compatibility conditions. They belong to the so-called infinite dimensional or distributed parameter systems. Such systems are usually solved numerically using finite element methods (FEM). Using this approach one can obtain results reflecting the reality rather accurately. FEM is an absolutely essential resource in solving problems in plant design, thermal and stress analysis, etc. However, computational effort increases dramatically depending on the accuracy required, especially for solving time dependent problems.

The approach used in model based control is to keep the models as simple as possible. This is done in order to be able to run the models on the limited computer hardware usually available at the growth furnaces. This is achieved by partitioning the system under consideration into domains which may reasonably well be considered as homogeneous, i.e., the material parameters which are assumed to be constant. Heat and mass transfer between these domains are calculated from appropriate mass and energy balance equations. Since spatial dependence of the parameters is neglected, one ends up with a set of ordinary differential equations. Such models are called finite dimensional or lumped parameter models. Using such models one can calculate the basic system behavior leading to a qualitatively and quantitatively sufficient insight into the process. Powerful methods for controller and observer design, trajectory planning, and dynamic analysis exist for this class of models. The lack of accuracy can be admitted by the introduction of feedback.

A global lumped parameter model of the overall Czochralski crystal growth system was first presented by Steel and Hill [59]. In order to simplify and improve parameterization of PID controllers they analyzed a linearized model in terms of a transfer function in several growth stages. The central idea of their approach consists in partitioning the Czochralski system into the four areas: melt - meniscus - crystal - ambient. This is still the basis of all lumped parameter models of the Czochralski process used to date. Using their method an a priori controller design could be dramatically improved, thus, avoiding time consuming empirical parameterization of PID controllers. However, this requires sufficient accuracy of the model.

Analysis of steady state conditions, the dynamics of the process, and optimal control design using lumped parameter models (in terms of a state space model) is subject of investigations presented by Satunkin and Rossolenko for the standard $\mathrm{Cz}$ process [20] as well as for the LEC process [60]. Considerations of optimal reference trajectory planning based on such models has been given by the same author in [61]. A compilation and extension of these results is presented in [31].

A very comprehensive and detailed model based analysis of the global Czochralski system is presented by Gevelber et al. [17, 43, 56, 57]. The first two publications of this series are based on a 7 th order lumped parameter model of the process while the latter ones make use of a more refined model.

Thorough analysis approaches based on distributed parameter models (treated for the quasi-stationary case) have been presented by Derby et al. [18, 19, 62, 63]. The great advantage of these approaches is the fact that the heat flow in the plant can be modeled quite accurately. However, much effort is necessary to adapt the models to the actual plant design and to determine the physical parameters needed in the model with sufficient accuracy.

\section{Approaches for control of the $\mathrm{Cz}$ process} 4.1. State estimation

In case of the Czochralski crystal growth the measurement of the crystal diameter, the primary variable to be controlled, is quite a complex task. Three different approaches exist: 
1. Optical capturing of meniscus shape, typically by detecting the bright meniscus ring [16, 41, 64, 65].

2. Evaluation of the force acting on a load cell mounted at the top of the pulling $\operatorname{rod}[15,32$, 34-36]. Also weighing of the crucible is possible $[66,67]$.

\section{Image processing of the whole crystal $[68,69]$.}

Optical imaging of the bright meniscus ring, which results from reflections of light emitted by the heaters, is the most important measuring technique used in silicon crystal growth. Here, one has to take into account that what is measured is the diameter of the meniscus at a certain height, not the crystal diameter at the three phase boundary. This means that the anomaly (cf. Sect. 2.2) comes into play: if the crystal radius begins to decrease, first the meniscus height will increase. This means that the diameter of the bright meniscus ring will increase which might result in incorrect reactions of the controller if it is not adapted to this behavior [17]. A detailed discussion on how to obtain an estimate of the real crystal diameter from the camera system is given in [70, 71].

In case of the liquid encapsulated Czochralski process where melt and meniscus are covered by a layer of boron oxide only the second technique can be applied. It is also often used when no camera system for the detection of the bright meniscus ring is available or applicable. It is based on the following idea: the gravitational force $F_{\mathrm{c}}$ of a rotationally symmetric crystal of length $l$ and density $\rho_{\mathrm{s}}$ can be calculated by integration of

$$
F_{\mathrm{c}}=\pi g \rho_{\mathrm{s}} \int_{0}^{l} r_{\mathrm{i}}^{2}(\lambda) \mathrm{d} \lambda
$$

with crystal radius $r_{\mathrm{i}}$ and gravitational acceleration $g$. Actually the force measured by the load cell is not equal to $F_{\mathrm{c}}$ in Eq. (9). In fact it is also influenced by forces resulting from the surface tension of the meniscus, the hydrostatic pressure of the melt raised over the melt level, and in case of LEC by the buoyancy forces resulting from the liquid boron oxide. For this reason things become considerably more complicated as it might seem from a first glance on Eq. (9). Details are discussed in [22].

The reconstruction of the crystal diameter and other quantities by means of an observer (cf. Sect. 3.4) is the subject of several publications. Satunkin and Leonov discuss the question of observability in case of $\mathrm{Cz}$ and LEC growth in [44]. The discussion is based on a linearized model of the overall process which is bound to the knowledge of some thermodynamical parameters. An elegant approach is presented in $[72,73]$ where, roughly speaking, an iterating algorithm is used which fits the theoretically calculated mass of the load cell to its real value by heuristically changing the crystal diameter in every step. However, this algorithm contains a parameter which must be empirically determined to guarantee convergence. A reduced order nonlinear tracking observer for the $\mathrm{Cz}$ and the LEC system including full meniscus dynamics is presented in [22]. As far as the system is tracked along its reference trajectory this approach produces very accurate results.

If the influence of the changing meniscus can be more or less neglected, a simple approach is presented in $[74,75]$. Here the basic idea is to exclude the meniscus dynamics from the model making it easy to solve the remaining equations for the radius. This method is useful for reconstruction of the radius during cylindrical growth and for crystals with slowly increasing diameter in the cone. If one is obliged to grow crystals with large slope angles in the shoulder this method fails, especially when fading into the cylinder.

The third method has no technological relevance because, on the one hand, it is very complicated to be realized and, on the other hand, because changes in growth are detected when they have already influenced the crystal (which is actually not the case when using the first and second technique where changes in the meniscus preceding a change in the crystal are detected) [76].

\subsection{Feed-forward control}

As described in Sect. 3.2, feed-forward control is an essential part of the overall control system. In crystal growth, a common method in practice is to determine the feed-forward control by careful analysis of repeated growth runs, resulting in a trajectory for the control inputs which can then be used as part of the recipe.

While this method is widely accepted it suffers from the fact that it is extremely time consuming and, thus, expensive, but functions if the same conditions are repeated and there are no significant variations from run to run. However, any change in plant setup or change in desired crystal cylinder diameter means repeating this procedure. Finally, not all details of the system dynamics can be determined by this empirical approach. If a sufficiently exact model of the process is available, this model can be used as a basis for determining proper feed-forward control trajectories which then can be fine-tuned in an empirical manner afterwards.

Such lumped parameter approaches are presented in [45] (4" silicon), [75] ( $\mathrm{InP})$, and [77]. Since the underlying models neglect the spatial distribution of system properties (cf. Sect. 3.5) most often some empirical corrections (e.g. in [75]) are introduced to fit the result to reality. An approach trying to circumvent this problem is proposed in [78], basing on a mathematical description regarding the most important qualitative dependences between the relative changes of manipulated and controlled variables during growth. For a certain thermal setup this approach allows to easily calculate reference trajectories for different crystal shapes and growth rates.

In order to overcome such limitations one may describe the system behavior without neglecting the spatial dependence of some of the system properties. Then one is confronted with the problem of numerically solving a set of time dependent $2 \mathrm{D} / 3 \mathrm{D}$ partial differential equations with free boundary conditions. Even though powerful finite element methods for solving such problems exist, and computational performance has dramatically 
increased in the last years this still is one of the most challenging tasks [79]. The situation gets even worse if feed-forward trajectories need to be rescheduled in real time during growth, for instance in order to react to some perturbations [80]. Extensive studies on this task can be found in [81-84], presented for the examples of growth of large diameter silicon and germanium crystals.

In order to reduce the computational effort one might restrict the problem to the quasi-stationary case. The feed-forward trajectory is then generated piecewise. This very powerful strategy has been presented in detail by Derby and Brown in [18, 19]. An approach using a simplified 2D-model especially designed for control purposes can be found in [85].

4.3. PID based control using optical diameter estimation

In Refs. [16, 41] a method for determining an estimate of the crystal radius is presented using optical imaging. The idea is based on the fact that light emitted by the red-hot heaters is reflected in upward direction from the meniscus. These reflections can be seen and detected by an optical camera as a so-called "bright ring". Then, image processing software is able to determine the diameter of the ring in real time. This value, scaled by an empirically determined factor accounting for the difference between measured meniscus and expected crystal radius, is used as the measured variable in the control system. Since silicon has a large Laplace constant (i.e., high surface tension and low density resulting in large meniscus heights) and because of its metallic reflectivity this material is an obvious candidate for this technique. Hence, although the crystal radius itself is not measured, this technique is widely and successfully used especially in silicon $\mathrm{Cz}$ growth [65, 86].

When designing a control system which tracks crystal diameter and growth rate (cf. Sect. 1) one has to reflect the different time constants the available control inputs have on the system: (i) Changes in pulling speed are affecting the system quite quickly; (ii) changes in heater power need some time depending on the thermal conditions occurring in the system $[17,43]$. Therefore, most often a cascaded control structure is chosen, assuming that pulling speed $v_{\mathrm{p}}$ is more or less equal to the growth rate (cf. Fig. 5) [87].

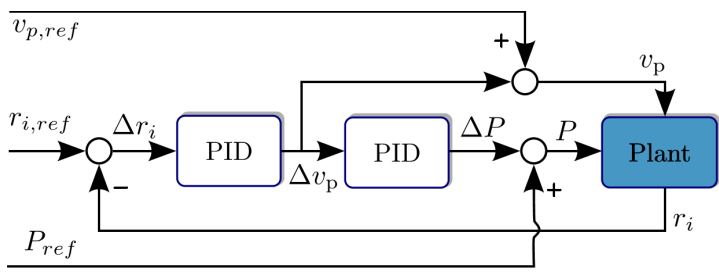

Fig. 5. Cascaded PID based control scheme utilizing pulling speed and heater power for diameter control (according to [87]).

Deviations $\Delta r_{\mathrm{i}}$ from the reference value $r_{\mathrm{i}, \mathrm{ref}}$ of the crystal radius $r_{\mathrm{i}}$ are fed into a PID controller manipulating the reference value $v_{\mathrm{p} \text {,ref }}$ of the pulling speed by an amount of $\Delta v_{\mathrm{p}}$. This value, indicating a deviation of the real pulling speed trajectory from its pre-calculated reference value, is also fed into a second PID controller manipulating the reference value $P_{\text {ref }}$ of the heater power by an amount $\Delta P$. By this strategy it is ensured that the system is able to react quite quickly on perturbations occurring in crystal diameter. Accumulated deviations in the pulling speed trajectory lead to a change of the heater power trajectory, ensuring that the system is kept on its reference state in a long term manner. Especially the pulling speed is brought back to its reference.

This control approach requires very careful planning of the feed-forward trajectories, especially for the pulling speed needed to obtain a certain growth rate trajectory.

\subsection{PID based control using weight measurement}

If one does not have the possibility to capture the diameter of the bright meniscus ring, the usual control strategy is to use the crystal or the crucible "weight" as measured variable $[15,88]$. The basic idea is to measure the force acting on the pulling or crucible rod and to compare it with the required force resulting from the reference shape of the crystal [32]. The deviation between both values is used to drive a PID controller manipulating the heater power. Crystal weighing is preferred due to problems of evaporation of material from the charge and the crucible in case of crucible weighing. When using rf-heaters additional vertical forces induced in the susceptor come into play also [86].

\subsubsection{Control system design}

In Fig. 6 the structure of a weight based control system is sketched. One has to distinguish between two modes of operation [86]:

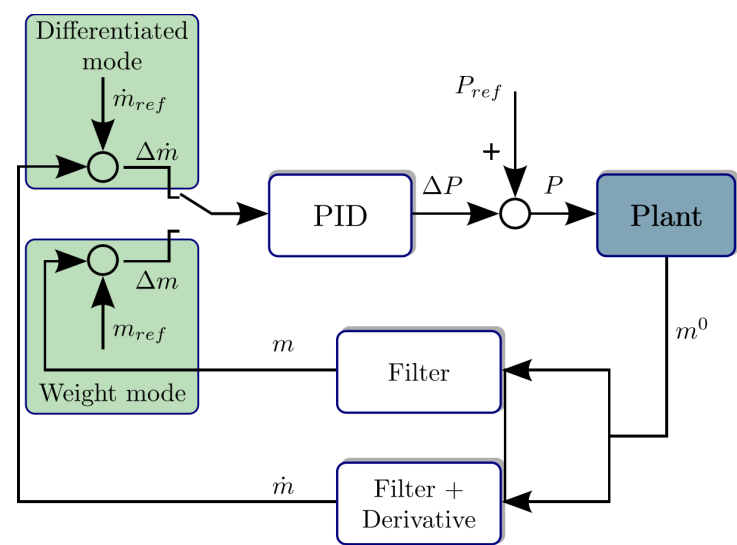

Fig. 6. Weight based PID control scheme utilizing heater power for diameter control.

Weight mode. The weight measured by the load cell is compared to the reference value and the difference is used as an error signal for the PID controller. Hence, in this mode the controller tries to keep the weight on its reference value, meaning that a previous error in crystal radius later results in an error of opposite sign. Thus, oscillations may occur. However, this mode circumvents 
noise generation by numerical differentiation of the force raw signal as required in differentiated weight mode.

Differentiated weight mode. The force raw signal is differentiated with respect to time and then compared to the reference value resulting from the predefined shape of the crystal. If one neglects the dynamics of the meniscus this signal is proportional to the crystal radius, i.e., this mode tries to keep the radius on its reference value without being influenced from the past. The differentiation of the raw signal leads to a phase advance in the control system which tends to make the system "more stable". On the other hand, sophisticated signal processing and filtering methods as well as high resolution load cells are required.

The most crucial part in this technique is the anomalous behavior of the signal, cf. Sect. 2.2. The consequence of this anomaly is that the performance of the control system is fundamentally limited. The controller "is only allowed to respond carefully" to any sort of perturbation since they could be of opposite direction not indicating what really goes on at the interface. For example, if the radius of the crystal increases, the controller would have to increase heater power in order to counteract this perturbation (i.e., the parameters $K_{\mathrm{P}}, K_{\mathrm{I}}, K_{\mathrm{D}}$ of the controller (5) must be chosen positive). However, what is first detected in this case is a decrease of the differentiated weight gain signal leading the controller to decrease heater power, thus countenancing the increase of the crystal radius.

\subsubsection{Improvements and optimizations}

In this section some approaches trying to compensate the problems mentioned above are sketched.

In Refs. [35, 36] Bardsley et al. present a sophisticated control algorithm containing a method trying to overcome the problems resulting from the anomalous behavior. They try to estimate the anomalous components in the measured weight gain signal and subtract them from the signal. The estimation is done using a linear model calculating the radius change $\delta r_{\mathrm{i}}$ induced by changes in heater power. This value can be used for calculating an estimate of the anomalous component of the signal. Experimental results presented in [33] demonstrate the performance of this approach. However, it is restricted to the knowledge of a sufficiently precise model of the process, especially of the thermal conditions in the furnace.

A challenging task in this control approach is the choice of the parameters of the PID controller. Most often this is done by trial and error. However, there exist some interesting approaches trying to determine optimal parameters of the PID controller on the basis of the model knowledge available. Satunkin et al. [20, 44] proposes an approach based on a cost functional for adjusting parameters in a single as well as in a multi-loop control system utilizing heater power and pulling speed as manipulated variables. The same authors present in [44] a modeling approach focusing on inaccuracies of the weight sensor and the consideration of these in the control system. Another model based approach can be found in [89].
As already mentioned the dynamic characteristic of the process changes during crystal growth. As a consequence parameters of the PID controller determined for one setpoint may not produce acceptable results as the process proceeds. The strategy to overcome this problem is to change the parameters on relevant intervals. This strategy is commonly known as parameter or gain scheduling in the field of adaptive control [90, 91]. An approach for $\mathrm{Cz}$ growth can be found in [89].

\subsection{Model based control}

\subsubsection{Lumped parameter model based control}

In Refs. [17, 43, 56, 57] Gevelber et al. propose a model based multi-loop control system. The first two publications of this series are based on a 7 th order model of the process, while the latter ones make use of a more refined model of order 34 . In these publications a multi-loop control system is proposed in order not only to ensure a correct diameter during the process, but also in order that identifiable disturbances are compensated before they affect the growth dynamics. Two main disturbances are identified, both related to the melt drop: the thermal state of the melt, which changes the heat flux entering the interface, and the thermal environment that the crystal sees, affecting the heat flux from the interface and thus the growth dynamics.

By adding, for instance, a closed loop around the melt temperature, the impact of this disturbance is significantly reduced, enabling tighter control of diameter and thus minimizing growth rate variations in a robust manner (e.g. not sensitive to model error or process variations). Additionally, this work analyzes the importance of controlling interface shape in addition to crystal diameter, in terms of meeting additional control objectives such as keeping thermal stress below a limit in order to minimize dislocation defects. Controlling interface shape in addition to diameter, requires the use of additional actuators, and an important part of the design task is to select an actuator set that can control both degrees of freedom relatively independently. To further obtain design insight Gevelber et al. derive a model which especially reflects the influence of radiation heat transfer within the furnace as exactly as possible within a lumped parameter model.

Another approach is presented in [21, 22]. There, the fact that it is easy to derive a sufficiently precise model of the pure meniscus dynamics in contrast to the fact that such a model of the thermal behavior is not available is utilized. Using a model of the meniscus dynamics a model based controller calculates desired values $v_{\mathrm{z}, \mathrm{ref}}$ of the ratio between pulling speed and growth rate quite similar to the approach presented in Sect. 3.3. Since one lacks a model of the thermal behavior, PID controllers are placed around this model based controller transforming this intermediate value into the inputs pulling speed $v_{\mathrm{p}}$ and heater power $P$ available at the process. This approach is sketched in Fig. 7. By this strategy a large amount of lumped parameter model knowledge is introduced into the control system. 


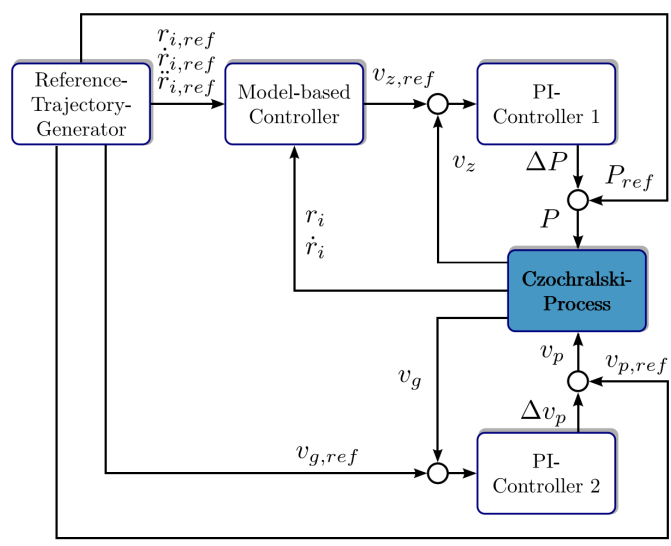

Fig. 7. Combination of model based and PID control according to [21, 22].

Further model based approaches can be found in [31] (using a linear state space controller), in [85] (using a 2D-approximation), and in [92] (combining heuristic and deterministic techniques in the $4^{\prime \prime}$ growth of GaAs crystals using the LEC method).

In all cases discussed in this section the overall closed loop performance is considerably enhanced, however, because of the lumped parameter character, some more or less strict constraints exist with respect to process setup, model validation or trajectory planning.

\subsubsection{Distributed parameter model based control}

Some publications try to overcome the limitations resulting from the lumped parameter approach by directly working on the partial differential equations of the distributed parameter models. Such approaches are for example presented by James $\mathrm{Ng}$ et al. in [93-95] and Wang in [96] and are fundamentally important for understanding the dynamics of the process, especially when focusing on the effects related to heat transport phenomena or variable phase boundary problems. However, for an implementation under real world conditions and controller parameterization they are not suitable. The reason for this is that in these models the manipulated variables are also spatially distributed, e.g. a temperature distribution on the heater surface. But: what the controller actually manipulates is the current entering the heater which is a lumped quantity which is in an unknown way connected to the temperature distribution on the heater surface.

\subsubsection{Model predictive control}

A third model based approach is the so-called model predictive control which is often used in process technology. Roughly speaking, model predictive control makes use of a dynamic model of the process, too. However, the way of calculating values for the manipulated variable is based on optimization methods, cf. Fig. 8. The solution of such optimization problems is well known and widely used in mathematics. For this purpose, in each time step the set of control activities recorded so far is evaluated in order to predict the values of the manipu- lated variable over a finite prediction horizon - based on the mathematical model and using optimization criteria. The optimization criteria, in some sense the "parameters" of a model predictive controller, reflect, for example, the dynamics of the deviation between desired and real values $[97,98]$. The advantage compared to the approaches presented in the previous section is that the underlying models can be arbitrarily complex. The disadvantage is the enormous computational effort. One has to keep in mind that for computing a new setpoint value of the manipulated variable several time dependent solutions of the model are required.

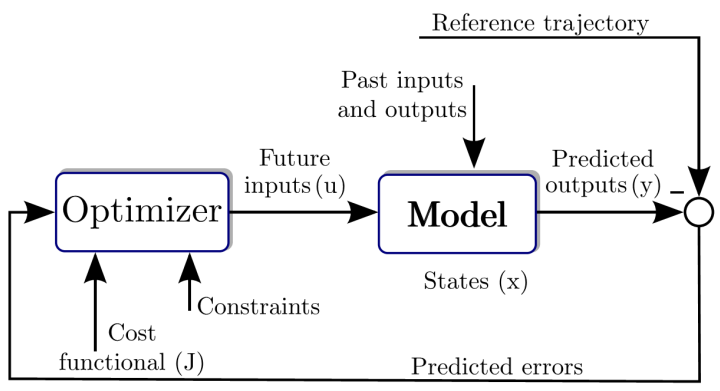

Fig. 8. Model predictive control.

An interesting approach based on this strategy can be found in [99, 100]. The model used in this approach is of lumped parameter type. It includes melt convection, which requires very strict assumptions to be fulfilled in order to make the model applicable. Another approach to establish such a model based control on the basis of a lumped parameter model has been published by Voronkov et al. [12, 87]. It is closely related to the $v_{\mathrm{g}} / G$ theory $[7,10]$ which requires very precise tracking of the growth rate $v_{\mathrm{g}}$.

\subsection{Further references}

So far, the challenges and some of the fundamental strategies for controlling the Czochralski crystal growth process have been presented. Unfortunately, because of the limited space available in this special issue, many details had to be skipped in the discussion. For this reason the reader is referred to much more exhaustive presentations of these subjects in [31, 73, 101-103].

\section{Conclusion}

The contribution gives a brief overview of selected strategies for the control of the Czochralski process. Although a large variety of approaches exist, the control of this process continues to be a challenging task employing scientists and engineers all around the world. By the very nature of the process, model based control is required to further improve yield and quality of the crystals. However, the choice of an appropriate model is crucial in this respect. These models must be simple enough and should yet cover the main dynamics. For the meniscus dynamics existing lumped parameter models are sufficient for very good control results. However, further progress may be 
expected from improved models of the thermal dynamics. These models should to a sufficient extent account for the spatially distributed nature of the heat transport phenomena, but they must still be simple enough to be useful for on-line control and estimation under industrial conditions.

\section{References}

[1] M. Cole, R. Ware, M. Whitaker, in: Proc. 5th Int. Conf. on Cryst. Growth, Boston 197r, 1977, Abstract 132 .

[2] D. Hurle, G. Joyce, M. Ghassempoory, A. Crowley, E. Stern, J. Cryst. Growth 100, 11 (1990).

[3] M. Neubert, P. Rudolph, Prog. Cryst. Growth Charact. Mater. 43, 119 (2001).

[4] S. Motakef, K. Kelly, K. Koai, J. Cryst. Growth 113 , 279 (1991).

[5] A. Jordan, R. Caruso, A. von Neida, J. Nielsen, J. Appl. Phys. 52, 3331 (1981).

[6] A. Jordan, A. von Neida, R. Caruso, J. Cryst. Growth 70, 555 (1984).

[7] V.V. Voronkov, J. Cryst. Growth 59, 625 (1982).

[8] M. Gevelber, G. Stephanopoulos, J. Cryst. Growth 84, 647 (1987).

[9] D. Hurle, in: Sir Charles Frank, OBE, FRS: An 80th Birthday Tribute, Eds. R. Chambers, J. Enderby, A. Keller, A. Lang, J. Steeds, Adam Hilger, Bristol 1991, p. 188.

[10] R. Falster, V. Voronkov, Mater. Res. Soc. Bull. 73, 28 (2000).

[11] M. Gevelber, D. Wilson, N. Duanmu, J. Cryst. Growth 230, 217 (2001).

[12] V. Voronkov, P. Mutti, US patent No's US 2002043206 and US 6726764 (2004).

[13] G. Teal, J. Little, Phys. Rev. 78, 647 (1950).

[14] L. Roth, W. Taylor, Proc. Inst. Radio Eng. 40, 1338 (1952).

[15] J. Levinson, US Patent No. 2,908,004, October 6th 1959 (1959).

[16] E. Patzner, R. Dessauer, M. Poponiak, Solid State Technol. 10, 25 (1967).

[17] M. Gevelber, M. Wargo, G. Stephanopoulos, J. Cryst. Growth 85, 256 (1987).

[18] J. Derby, R. Brown, J. Cryst. Growth 74, 605 (1986).

[19] J. Derby, R. Brown, J. Cryst. Growth 75, 227 (1986).

[20] G. Satunkin, S. Rossolenko, Cryst. Res. Technol. 21, 1125 (1986).

[21] J. Winkler, M. Neubert, J. Rudolph, J. Cryst. Growth 312, 1005 (2010).

[22] J. Winkler, M. Neubert, J. Rudolph, J. Cryst. Growth 312, 1019 (2010).

[23] J. Mullin, B. Straughan, W. Brickell, J. Phys. Chem. Solids 26, 782 (1965).

[24] D. Looze, A. Farzin, B. Bernstein, J. Cryst. Growth 148, 79 (1995).

[25] A. Jordan, R. Caruso, A. von Neida, Bell System Tech. J. 62, 477 (1983).

[26] T. Johansen, J. Cryst. Growth 80, 343 (1987).

[27] K. Azuma, Jap. Patent No. 60-11299 (1983).
[28] P. Rudolph, M. Neubert, S. Arulkumaran, M. Seifert, Cryst. Res. Technol. 32, 35 (1997).

[29] M. Neubert, P. Rudolph, Mitteilungsblatt der DGKK 69, 11 (1999) (in German).

[30] Crystal Growth Techniques Based on Capillary: Czochralski, Floating Zone, Shaping and Crucible Process, Ed. T. Duffar, Wiley, Hoboken 2010.

[31] G. Satunkin, Prog. Cryst. Growth Character. Mater. 56, 1 (2010).

[32] W. Bardsley, G. Green, C. Holliday, D. Hurle, J. Cryst. Growth 16, 277 (1972).

[33] W. Bardsley, F. Frank, G. Green, D. Hurle, J. Cryst. Growth 23, 341 (1974).

[34] W. Bardsley, B. Cockayne, G. Green, D. Hurle, G. Joyce, J. Roslington, P. Tufton, H. Weber, J. Cryst. Growth 24/25, 369 (1974).

[35] W. Bardsley, D. Hurle, G. Joyce, J. Cryst. Growth 40, 13 (1977).

[36] W. Bardsley, D. Hurle, G. Joyce, G. Wilson, J. Cryst. Growth 40, 21 (1977).

[37] E. Boucher, T. Jones, J. Chem. Soc. Faraday Trans. 176, 1419 (1980).

[38] K. Mika, W. Uelhoff, J. Cryst. Growth 30, 9 (1975).

[39] T. Surek, S. Coriell, B. Chalmers, J. Cryst. Growth 50, 21 (1980).

[40] J. Brice, J. Cryst. Growth 2, 393 (1968).

[41] T. Digges, R. Hopkins, R. Seidensticker, J. Cryst. Growth 29, 326 (1975).

[42] T. Johansen, J. Cryst. Growth 118, 353 (1992).

[43] M. Gevelber, G. Stephanopoulos, M. Wargo, J. Cryst. Growth 91, 199 (1988).

[44] G. Satunkin, A. Leonov, J. Cryst. Growth 102, 592 (1990).

[45] K. Kim, A. Kran, P. Smetana, G. Schwuttke, J. Electrochem. Soc. 130, 1156 (1983).

[46] N. Duanmu, Ph.D. Thesis, College of Engineering, Boston University, 2006.

[47] T. Johansen, J. Cryst. Growth 84, 609 (1987).

[48] T. Johansen, J. Cryst. Growth 123, 188 (1992).

[49] T. Surek, J. Appl. Phys. 47, 4384 (1976).

[50] V. Tatarchenko, E. Brener, J. Cryst. Growth 50, 33 (1980).

[51] G. Babkin, E. Brener, V. Tatarchenko, J. Cryst. Growth 50, 45 (1980).

[52] D. Hurle, J. Cryst. Growth 128, 15 (1993).

[53] J. Derby, R. Brown, J. Cryst. Growth 83, 137 (1987).

[54] A. Crowley, IMA J. Appl. Math. 30, 173 (1983).

[55] T. Johansen, J. Cryst. Growth 114, 27 (1991).

[56] M. Gevelber, J. Cryst. Growth 139, 286 (1994).

[57] M. Gevelber, J. Cryst. Growth 139, 271 (1994).

[58] D.G. Luenberger, IEEE Trans. Milit. Elec. 8, 74 (1964).

[59] G. Steel, M. Hill, J. Cryst. Growth 30, 45 (1975).

[60] G. Satunkin, J. Cryst. Growth 154, 172 (1995).

[61] G. Satunkin, S. Rossolenko, V. Kurlov, B. Reg'kin, V. Tatarchenko, A. Avrutik, Cryst. Res. Technol. 21, 1257 (1986). 
[62] J. Derby, R. Brown, J. Electrochem. Soc. 132, 470 (1985).

[63] P. Thomas, J. Derby, L. Atherton, R. Brown, J. Cryst. Growth 96, 135 (1989).

[64] U. Gross, R. Kersten, J. Cryst. Growth 15, 85 (1972).

[65] R. Lorenzini, F. Nuff, D. Blair, Solid State Technol. 2, 33 (1974).

[66] A. Zinnes, B. Nevis, J. Cryst. Growth 19, 187 (1973).

[67] A. Valentino, C. Brandle, J. Cryst. Growth 26, 1 (1974).

[68] H. van Dijk, C. Jochem, G. Scholl, P. van der Werf, J. Cryst. Growth 21, 310 (1974).

[69] K. Bachmann, H. Kirsch, K. Vetter, J. Cryst. Growth 7, 290 (1970)

[70] S. Kimbel, Ph.D. Thesis, Washington University, 2000.

[71] S. Kimbel, J. O'Sullivan, Proc. SPIE 4188, 45 (2001).

[72] S. Rossolenko, I. Pet'kov, V. Kurlov, B. Red'kin, J. Cryst. Growth 116, 185 (1992).

[73] N. Abrosimov, V. Kurlov, S. Rossolenko, Prog. Cryst. Growth Charact. Mater. 46, 1 (2003).

[74] E. Kubota, Cryst. Res. Technol. 34, 539 (1999).

[75] M. Masi, S. Carra, M. Polli, M. Ratti, G. Guadalupi, Mater. Chem. Phys. 66, 236 (2000).

[76] G. Joyce, D. Hurle, Q. Vaughan, J. Cryst. Growth 132, 1 (1993).

[77] H. Nalbandyan, J. Cryst. Growth 67, 115 (1984).

[78] M. Neubert, J. Winkler, "Nonlinear Model-Based Control of the Czochralski Process IV: Feedforward Control", J. Cryst. Growth, submitted for publication.

[79] G. Brown, T. Kinney, P. Sackinger, D. Bornside, J. Cryst. Growth 97, 99 (1989).

[80] A. Voigt, M. Metzger, "Numerical Simulation and Control of Industrial Crystal Growth by the Czochralski and Vertical Gradient Freeze Method", Caesar Preprint, August 2000.

[81] E. Dornberger, W. von Ammon, N. van den Bogaert, F. Dupret, J. Cryst. Growth 166, 452 (1996).

[82] N. van den Bogaert, F. Dupret, J. Cryst. Growth 171, 65 (1997).

[83] N. van den Bogaert, F. Dupret, J. Cryst. Growth 171, 77 (1997).

[84] E. Olivari, P. Jacmin, N. van den Bogaert, V. Wertz, F. Dupret, J. Cryst. Growth 180, 627 (1997).
[85] A. Sabanskis, K. Bergfelds, A. Muiznieks, T. Schröck, A. Krauze, J. Cryst. Growth 337, 9 (2013).

[86] D. Hurle, J. Cryst. Growth 42, 473 (1977).

[87] V. Voronkov, P. Mutti, U.S. Patent No. US 2002/0043208 A1 (2002).

[88] T. Rummel, Apparatus for pulling rod-shaped crystals of semiconductor material from a melt in a crucible, US Patent No. 3,259,467, July 5th 1966.

[89] M. Neubert, J. Winkler, J. Cryst. Growth 360, 3 (2012).

[90] K.J. Åström, B. Wittenmark, Adaptive Control, Prentice Hall, Upper Saddle River (NJ) 1994, Ch. 9.

[91] Industrial Digital Control Systems, Vol. 37 of IEEE Control Engineering Solutions, Eds. K. Warwick, D. Rees, Peter Peregrinus Ltd., Stevenage 1988, Ch. 14.7 .

[92] K. Riedling, J. Cryst. Growth 89, 435 (1988).

[93] J. Ng, S. Dubljevic, J. Proc. Contr. 21, 1369 (2011).

[94] J. Ng, S. Dubljevic, Chem. Eng. Sci. 67, 111 (2012).

[95] J. Ng, I. Aksikas, S. Dubljevic, Int. J. Contr., 1 (2013).

[96] P. Wang, Optim. Contr. Appl. Meth. 16, 305 (1995).

[97] Nonlinear Model Predictive Control, Vol. 26 of Progress in Systems and Control Theory, Eds. F. Allgöwer, A. Zheng, Birkhäuser Verlag, Basel 2007.

[98] E. Camacho, C. Bordons, Model Predictive Control, Advanced Textbooks in Control and Signal Processing, Springer, Berlin 2004.

[99] R. Irizarry-Rivera, W.D. Seider, J. Cryst. Growth 178, 593 (1997).

[100] R. Irizarry-Rivera, W.D. Seider, J. Cryst. Growth 178, 612 (1997).

[101] J. Winkler, M. Neubert, J. Rudolph, N. Duanmu, M. Gevelber, Crystal Growth Processes Based on Capillarity, Wiley, 2010, p. 115.

[102] Y.A. Tatarchenko, Shaped Crystal Growth, Vol. 20 of Fluid Mechanics and its Applications, Kluwer Academic, Dordrecht 1994.

[103] Handbook of Crystal Growth, Vol. 2: Bulk Crystal Growth, Ed. D.T.J. Hurle, North Holland Elsevier, Amsterdam 1994 